\title{
Behavioural disorders in children with epilepsy: early improvement after surgery
}

\author{
M Lendt, C Helmstaedter, S Kuczaty, J Schramm, C E Elger
}

\begin{abstract}
Objectives-Epilepsy surgery has proved to be a successful intervention method to achieve freedom from seizures or seizure relief in children with pharmacoresistant epilepsy. Long term studies on operated children suggest that behavioural disorders, which are often seen before surgery, improve after surgery. However, the early postoperative development of behavioural problems has not been systematically evaluated.
\end{abstract}

Methods-Parents of 28 children with pharmacoresistant focal epilepsies completed the child behaviour checklist (CBCL) preoperatively and 3 months after surgery. Surgeries comprised 24 focal resections (13 temporal, 11 extratemporal), two hemispherectomies, and two callosotomies. Twenty eight conservatively treated children with comparable CBCL scores served as a control group. A repeated measurement multivariate analysis of variance (MANOVA) and a regression analysis were computed to compare the development of behaviour between both groups and to identify predictors of postoperative changes in behaviour.

Results-Preoperatively $39 \%$ of the children exhibited significant behavioural problems, a further $11 \%$ were within the borderline range. The MANOVA disclosed a significant interaction between time of examination and group $(F=2.23, p<0.05)$. The surgery group showed significant improvements on the scales "internalising problems", "externalising problems", "attention problems", and "thought problems". Behavioural problems in the control group, however, remained unchanged. No changes were seen in social problems in both groups. The significant predictor of total behavioural improveEnileptology, Sigmund-Freud-Strasse 25, 53105 Bonn, Germany

$M$ Lendt

C Helmstaedter

S Kuczaty

C E Elger

Department of Neurosurgery

J Schramm

Correspondence to:

Dr M Lendt

psych@mailer.meb.uni-bonn.de

Received 19 April 2000 and in revised form

17 July 2000

Accepted 7 August 2000
Epilepsy in children is often accompanied by behavioural disorders. ${ }^{1-3}$ In an epidemiological study, McDermott et al found a 4.7 times higher prevalence of behavioural disorders in children with epilepsy compared with healthy children. The prevalence is also higher than in children with other chronic illnesses such as cardiac disease or diabetes. ${ }^{23}$

Behavioural disorders that have been consistently seen in children with epilepsy are hyperactivity, attention disorders, social withdrawal, conduct problems, and aggression. ${ }^{2-5}$ There is a general consensus that behavioural disorders are multiaetiological. ${ }^{67}$ Hermann et al showed that biological, psychosocial, demographic, and medication factors contribute to behaviour disorders. With regard to the epilepsy, a high seizure frequency and an early onset have been identified as most important risk factors. ${ }^{6-8}$ Whether the occurrence or extent of behavioural disorders depends on the seizure type, is largely unknown. There is some evidence that children with symptomatic focal epilepsies, especially those with a temporal focus, exhibit a particular high risk of behavioural disorders. ${ }^{159}$ However, the relevance of aetiology and seizure type diminishes when seizure frequency, age at onset, sex, and psychosocial variables are considered as covariates within multiple regression analyses. ${ }^{67}$

The influence of epilepsy surgery on behavioural disorders has been evaluated for different types of surgical interventions. Most of the studies have focused on the behavioural development after temporal lobe resection (TLR); some studies have also dealt with resections in other cortical areas. ${ }^{810} 11$ Davidson and Falconer evaluated the outcome of 40 children with follow up intervals up to 24 years after TLR. They found a normalisation of behaviour in $50 \%$ of the children and an improvement in $14 \%$ of the sample. ${ }^{11}$ Lindsay et al reported that $88 \%$ of their samples, which consisted mainly of children with temporal lobe epilepsy, showed psychiatric disturbances before surgery. At follow up, more than 5 years after surgery in most patients, improvements in social behaviour were seen, and no patient required psychiatric treatment. ${ }^{10}$

Studies suggest a particularly good outcome in patients who underwent hemispherectomy or callosotomy. ${ }^{12-14}$ Wilson's study on 50 hemispherectomy patients showed a normalisation in $93 \%$ of the children with preoperative behavioural disorders and no child showed a deterioration in behaviour. ${ }^{12}$ Behavioural outcome after callosotomy in children was documented by Lassonde and Sauerwein, who found improvements in social adjustment in $88 \%$ of their sample. ${ }^{14}$ 
Table 1 Subject characteristics

\begin{tabular}{|c|c|c|c|}
\hline & $\begin{array}{l}\text { Surgery group } \\
(n=28)\end{array}$ & $\begin{array}{l}\text { Control group } \\
(n=28)\end{array}$ & $\begin{array}{l}\text { Group } \\
\text { difference }\end{array}$ \\
\hline \multicolumn{4}{|l|}{ Age (y) at baseline examination: } \\
\hline Mean (SD) & $11.5(3.5)$ & $10.3(3.7)$ & $\mathrm{NS}^{\star}$ \\
\hline Range & $5-16$ & $4-16$ & \\
\hline \multicolumn{4}{|l|}{ Age (y) at onset of epilepsy: } \\
\hline Mean (SD) & $5.6(4.2)$ & $5.4(4.3)$ & $\mathrm{NS}^{\star}$ \\
\hline Range & $0-15$ & $0-15$ & \\
\hline IQ (mean $(\mathrm{SD})$ ) & $80.7(16.8)$ & $84.2(22.8)$ & $N S^{\star}$ \\
\hline Range & $50-115$ & $54-119$ & \\
\hline Sex (male/ female) & $14 / 14$ & $18 / 10$ & NSt \\
\hline Seizure type (SPS/CPS/SGS, drop attacks) & $2 / 17 / 9$ & $1 / 22 / 5$ & NSt \\
\hline \multicolumn{4}{|l|}{ Seizure frequency (seizures/month): } \\
\hline Median & 17.5 & 30.0 & NS $\ddagger$ \\
\hline Seizure outcome & & & $\mathrm{p}<0.001 \dagger$ \\
\hline Seizure free & $21(75 \%)$ & $4(14 \%)$ & \\
\hline Sign $(\geqslant 50 \%)$ reduction in seizure frequency & $5(18 \%)$ & $6(22 \%)$ & \\
\hline Seizue frequency unchanged & $2(7 \%)$ & $18(64 \%)$ & \\
\hline Baseline medication & & & NS† \\
\hline No medication & 1 & 1 & \\
\hline Monotherapy & 3 & 8 & \\
\hline Polytherapy & 24 & 19 & \\
\hline Antiepileptic treatment & & & NSt \\
\hline Number of drugs increased & 2 & 5 & \\
\hline Number of drugs reduced & 11 & 5 & \\
\hline Number of drugs unchanged & 15 & 18 & \\
\hline
\end{tabular}

SPS=Single partial seizures; CPS=complex partial seizures; $S G S=$ secondary generalised seizures; ${ }^{\star} t$ test; $\dagger \chi^{2}$ test; $\ddagger$ Mann-Whitney $U$ test $(\mathrm{p}>0.05)$.

Taking these findings into consideration, the data suggest a favourable development of behavioural disorders in children after different types of neurosurgical interventions in medically intractable epilepsies. However, there is legitimate criticism that these studies may overemphasise behavioural outcome after surgery. Firstly, the methods of assessment of behaviour were not documented in these investigations. Secondly, the follow up intervals varied greatly within each sample. Long follow up intervals, 24 years for example, in the study of Davidson and Falconer, increase the probability that factors other than surgery have influenced behavioural development. ${ }^{11}$ Thirdly, all studies lack a control group to exclude medication effects, although changes in drug regimen have repeatedly shown an influence on behaviour in children and adults. ${ }^{15} 16$

The aim of the present study was to systematically investigate the early changes in behaviour of children after epilepsy surgery in comparison with a control group of children who were solely medically treated. We evaluated the behavioural status before and 3 months after surgery by standardised parents' questionnaire. In addition, we analyzed whether changes in behaviour can be predicted on the basis of preoperatively available information.

\section{Patients and methods}

PATIENTS

Fifty six patients were included in this study. Twenty eight children underwent epilepsy surgery, 28 were treated conservatively. Criteria for inclusion were the careful diagnosis of focal epilepsy, pharmacoresistance since at least 1 year, and an age between 4 and 16 years. Diagnosis of epilepsy was made on the basis of seizure semiology, interictal EEG, and ictal EEG in all patients of the surgery group and most patients of the control group. All children underwent high resolution MRI. Children with pseudoseizures were excluded from this study.
Surgery group

We assessed 28 children between the ages of 5 to 16 years, who consecutively underwent presurgical evaluation between October 1996 and June 1999 in the Department of Epileptology, and were operated on in the Department of Neurosurgery, University of Bonn. Criteria for inclusion in the study were a surgical intervention performed to achieve seizure control, and a follow up examination 3 months after surgery.

Surgery consisted of 24 focal resections, two hemispherectomies, and two callosotomies. Most focal resections were located in the temporal lobes $(n=13)$, followed by the parietooccipital lobes $(n=7)$ and the frontal lobes $(n=4)$. Types of surgery were pure lesionectomies $(n=10)$, lesionectomies plus selective amygdalohippocampectomy $(\mathrm{n}=4)$, lesionectomy plus subpial transections $(n=4)$, or pure selective amygdalohippocampectomies $(n=6)$. Surgery was equally distributed in the left $(n=13)$ and in the right hemispheres $(n=13)$. Histopathological examination of 26 patients disclosed cortical dysplasia in nine, low grade tumours in seven, and hippocampal sclerosis in six patients, and gliosis, necrosis, angiomatosis, and haematoma in one patient each.

Antiepileptic drug treatment before surgery was a polytherapy in most children. Twenty children received a combination of a standard antiepileptic drug (carbamazepine etc) and one of the so called "new" antiepileptic drugs (vigabatrin, lamotrigine etc). Drug schedules remained unchanged between presurgical evaluation and postoperative follow up in 15 patients. Eleven patients were put on monotherapy. In two children an antiepileptic drug was added preoperatively to optimise postoperative seizure protection (table 1 ).

\section{Control group}

The control group consisted of 28 children with focal epilepsy who were consecutively admitted to our hospital between May 1997 and April 1999. One part of this group had undergone presurgical evaluation, but was not operated on, because no focal seizure origin could be identified. The second part of this group were children who had been admitted to our hospital to start a further treatment attempt with one of the new antiepileptic drugs. The control group did not differ from the surgical group on any of the demographical data taken (age, sex, IQ), seizure characteristics (type and frequency of seizures), and number of drugs at baseline assessment (table 1). Brain MRI evidence of a lesion was found in 18 patients (64\%). Although not significant, changes in medication schedule differed between both groups. Compared with the surgical group less children in the control group had a withdrawal of a drug and more children in the control group had an increase of drugs. Seizure outcome was significantly better in the surgical group than in the control group $\left(\chi^{2}=24.45\right.$, $\mathrm{p}=0.001)$. 
Table 2 Mean CBCL scores of both groups at baseline and follow up

\begin{tabular}{|c|c|c|c|c|c|c|}
\hline \multirow[b]{2}{*}{$C B C L$ scale } & \multicolumn{3}{|c|}{ Surgery group } & \multicolumn{3}{|l|}{ Control group } \\
\hline & $\begin{array}{l}\text { Baseline } \\
\text { Mean (SD) }\end{array}$ & $\begin{array}{l}\text { Follow up } \\
\text { Mean }(S D)\end{array}$ & F test & $\begin{array}{l}\text { Baseline } \\
\text { Mean (SD) }\end{array}$ & $\begin{array}{l}\text { Follow up } \\
\text { Mean }(S D)\end{array}$ & F Test \\
\hline Internalising problems & $59.7(9.9)$ & $56.3(10.6)$ & $\mathrm{p}=0.001$ & $56.8(10.6)$ & $58.4(11.0)$ & NS \\
\hline Externalising problems & $54.8(9.0)$ & $50.3(10.1)$ & $\mathrm{p}=0.002$ & $52.4(10.7)$ & $54.5(9.6)$ & NS \\
\hline Social problems & $60.6(9.7)$ & $59.4(8.4)$ & NS & $60.7(9.7)$ & $62.6(10.5)$ & NS \\
\hline Thought problems & $58.5(9.7)$ & $53.6(6.6)$ & NS & $55.0(7.9)$ & $57.0(7.7)$ & NS \\
\hline Attention problems & $61.6(9.1)$ & $58.3(6.9)$ & $\mathrm{p}=0.014$ & $61.2(8.2)$ & $62.3(9.7)$ & NS. \\
\hline
\end{tabular}

$F$ tests refer to univariate pre-post comparison for each group separately.

ASSESSMENT OF BEHAVIOUR

To assess the behaviour of the children their parents completed the child behaviour checklist (CBCL). ${ }^{17}{ }^{18}$ The CBCL is an internationally established instrument for the assessment of behavioural disorders and has proved validity in children with epilepsy. ${ }^{6}{ }^{19}$ The questionnaire consists of 113 questions on problematic behaviour in children. It provides a general behavioural problem index (total score) and scores on different subscales. According to previous research on behavioural problems in children with epilepsy we used the scales "internalising behaviour problems", "externalising behaviour problems", "social problems", "thought problems", and "attention problems". ${ }^{20}$ The scales internalising behaviour problems and externalising behaviour problems are derived from second order factorial analyses. They represent overcontrolled behavioural problems (social withdrawal, somatic complaints, depression) and under-controlled behaviour problems (aggression, delinquent behaviour). Behavioural problems are measured by $T$ scores. Scores equal to or less than 50 represent few or no behavioural problems, whereas the highest score 100 indicates extensive behavioural disorders. Cut off scores on the scales also allow a classification of a child into the categories "normal", "borderline" or "behaviourally disordered".

Parents of the surgical group were requested to answer the questionnaire at the time of presurgical evaluation and also at the standard follow up examination in our hospital 3 months after surgery. Because surgery was not always performed immediately after the presurgical evaluation, the interval between preoperative and postoperative evaluation ranged between 3 and 10 months (mean 5.3 (SD 1.8) months).

Baseline assessment of the control group was conducted at the time of admission to our hospital. Data from the follow up evaluation were gathered within the routine examination in our hospital or by mail. The duration of the follow up interval was 6.2 (SD 3.0) months, which matches that of the surgical group $(t=1.47$, $\mathrm{p}>0.05)$. All CBCL questionnaires were answered during the first and the second examination by the same parent.

DATA ANALYSIS

Firstly, changes in behavioural scores were analyzed by a repeated measurement MANOVA with group (surgical group/control group) as between factor and time of assessment (baseline/follow up) as within factor. As changes in antiepileptic drug regimen, particu- larly changes in the number of antiepileptic drugs, have been shown to influence cognition as well as behaviour in patients, changes in number of drugs (increase/reduction/no change) were considered as a covariates. ${ }^{15} 1621$ Secondly, we evaluated postoperative changes in the patient's total score classification-that is, the assignment to the categories "normal" ( $T$ between 50 and 59), "borderline" ( $T$ between 60 and 63), or "disordered" ( $T$ higher than 63), by means of the Wilcoxon ranks test. Finally, we analyzed potential predictors of changes in behaviour of the surgery group by a multiple regression analyses, which included sex, age at surgery, onset and duration of epilepsy, CBCL baseline score, side and site of the focus, seizure outcome as independent variables, and changes in behaviour (difference scores) as dependent variables.

\section{Results}

SURGERY GROUP VERSUS CONTROL GROUP

Changes in CBCL group means

Baseline analyses disclosed the highest CBCL scores for both groups on the scales attention problems and social problems followed by internalising behaviour problems, thought problems, and externalising behaviour problems (table 2). Baseline mean scores did not differ significantly between surgical group and control group, neither in multivariate statistics (Hotelling's $F=0.764, \mathrm{p}>0.05$ ) nor in univariate $F$ tests of the five scales ( $F$ between 0.001 and 2.114, p between 0.152 and 0.978).

The repeated measurement MANOVA with group (surgical group/control group) as between factor, time of assessment (baseline/ follow up) as within factor, and changes in number of drugs as covariate showed a significant interaction between time of assessment and group (Hotelling's $F=4.124, \mathrm{p}=0.003$ ). The mean CBCL scores of the surgical group decreased at follow up, whereas the scores of the control group slightly increased (table 2). Neither the main effects (time of assessment: Hotelling's $F=1.137, \mathrm{p}>0.05$, group: Hotelling's $F=0.576, p>0.05$ ), nor the interaction between time of assessment and the covariate changes in number of drugs became significant (Hotelling's $F=0.608, \mathrm{p}>0.05$ ).

Univariate analyses showed significant interaction effects between time of assessment and group on four CBCL scales. At follow up, the surgical group showed significantly lower scores than the control group on the scales internalising behaviour problems $(F=9.54$, $\mathrm{p}=0.003$ ), externalising behaviour problems $(F=11.76, \quad$ p=0.001), thought problems 
$50.0 \%$

Baseline

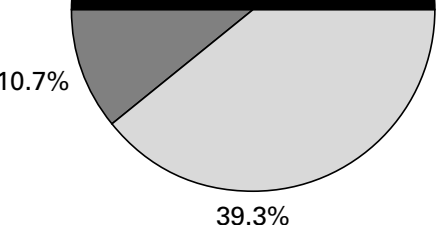

$39.3 \%$

Follow up

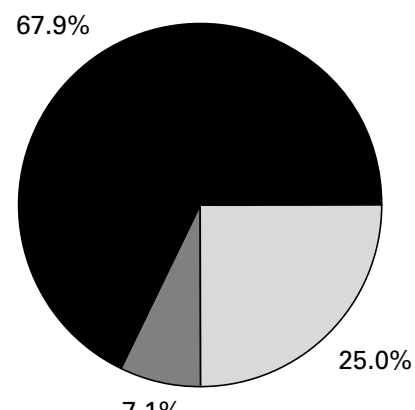

$7.1 \%$

Surgery group
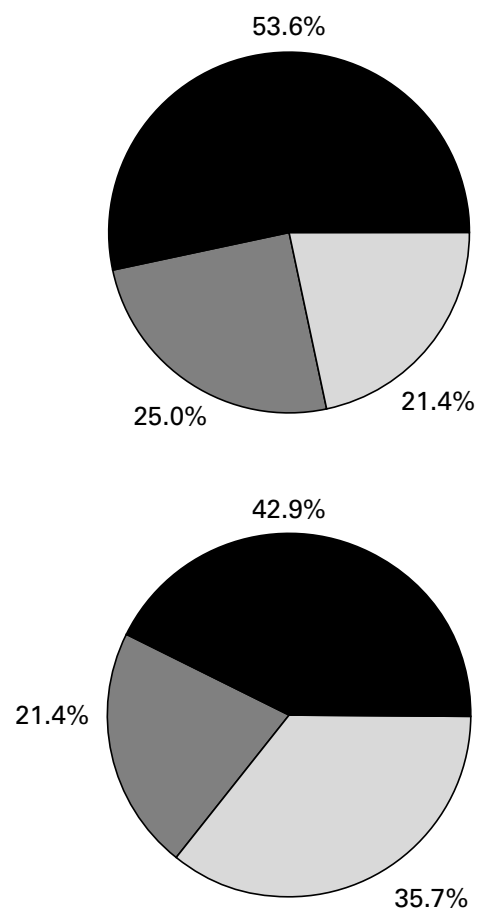

Control group changed in a positive sense in the surgical group $(Z=-2.10, \mathrm{p}<0.05)$. Three children exhibiting disordered behaviour preoperatively had a postoperative normalisation of behaviour and another two children had borderline scores. Of those three patients in the category borderline, two showed a normalisation, and only one child moved to the category disordered. By contrast with the surgical group the classification of control group tended to become worse at follow-up ( $Z=-1.96$, $\mathrm{p}=0.05)$. Only one child of the category disordered changed to borderline, but four children of the category borderline moved to the category disordered. In addition, three children classified as normal at baseline were classified in lower categories at follow up.

\section{RELATIONS BETWEEN CHANGES IN DIFFERENT} BEHAVIOURAL DOMAINS

Correlational and factorial studies of the CBCL subscales have shown high interrelations between the subscales. ${ }^{17}{ }^{18}$ To analyze the pattern of behavioural changes in the surgical group, we computed correlations among CBCL change scores (postoperative minus preoperative scores). Significant positive relations were evident between changes in attention problems and changes on the remaining four CBCL scales ( $r$ between 0.34 and $0.57, \mathrm{p}$ between 0.04 and 0.001 , one tailed significance). Furthermore, changes in externalising borderline or disordered (fig 1). At follow up examination the classification significantly 
Table 3 Correlations between change scorest of CBCL scales in the surgical group

\begin{tabular}{|c|c|c|c|c|}
\hline & $\begin{array}{l}\text { Internalising } \\
\text { problems }\end{array}$ & $\begin{array}{l}\text { Externalising } \\
\text { problems }\end{array}$ & Social problems & $\begin{array}{l}\text { Thought } \\
\text { problems }\end{array}$ \\
\hline Internal problems & - & & & \\
\hline External problems & 0.23 & - & & \\
\hline Social problems & 0.24 & $0.67 \star \star$ & - & \\
\hline Thought problems & 0.21 & 0.04 & 0.18 & - \\
\hline Attention problems & $0.40^{\star}$ & $0.34^{\star}$ & $0.36^{\star}$ & $0.57 \star \star$ \\
\hline
\end{tabular}

${ }^{\star} \mathrm{p}<0.05 ;{ }^{\star} \mathrm{p}<0.01$ (Pearson correlations, one tailed significance); †change score: postoperativepreoperative $T$ score.

Increase of

behavioural problems

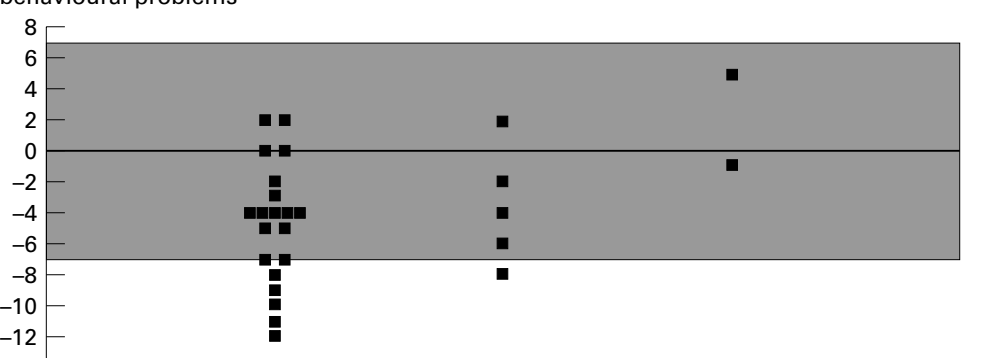

$-12-$

$-14$

$-16-$

$-18$

Decrease of Seizure free Reduction in seizure Seizure frequency

behavioural problems

frequency $(>50 \%) \quad$ unchanged

Figure 2 Relation between changes in behaviour and postoperative seizure outcome. $y$ Axis indicates difference scores of CBCL total scores (postoperative minus preoperative), $x$ axis represents seizure outcome.

behaviour problems were correlated with changes in social problems $(r=0.67, \mathrm{p}=0.001$; table 3).

PREDICTORS OF BEHAVIOURAL CHANGES

To find possible predictors of changes in behaviour in the surgical group we computed a stepwise multiple regression analyses with changes in CBCL scores (postoperativepreoperative scores) as dependent variable. As postoperative improvements in behaviour were significant on three of the five CBCL subscales and the direction of changes was uniform on all five scales, we used the total score instead of the subscores for this analysis. The group of independent variables included CBCL total score at baseline, age at surgery, sex, age at onset, duration of epilepsy, site of the focus (temporal $v$ extratemporal), side of the focus (left $v$ right hemisphere), and seizure outcome (seizure free/ reduction in seizure frequency/ seizure frequency unchanged).

Changes in the total behaviour score were significantly predicted by seizure outcome (adjusted $R^{2}=0.11, \mathrm{p}=0.047$ ), but were not related to any further variable ( $T$ between 0.24 and -0.33 , p between 0.99 and 0.75$)$. The better the seizure outcome, the higher was the postoperative reduction in global behaviour problems (fig 2).

\section{Discussion}

Our study confirms the high prevalence of behaviour problems in children with medically intractable epilepsies. ${ }^{81012}$ In $50 \%$ of our surgical sample the preoperative behaviour was classified as clinically disordered or at least borderline. Behavioural problems were particularly found in the domains of attention and social problems. The surgical intervention, however, led to a marked improvement of behavioural problems. Five children with high indices of behaviour disorders even showed a postoperative normalisation of behaviour. Improvements were found in internalising (overcontrolled) and externalising (undercontrolled) behaviour, attention problems, and, compared with the development of non-operated children with similar epilepsy conditions, also in thought problems. Thus, the parents reported improvements in many behaviour domains, but they did not describe postoperative changes in the social problems of the children. The scale social problems largely measures problems with peers (for example, social isolation, stigma), thus indicating that the social integration of the children is not improved in the first postoperative months despite freedom of seizures or seizure relief. This points out impressively the difficulties faced by operated children, which probably result from the persistence of negative attitudes in their social environment.

Although postoperative improvements in behaviour disorders have been described in earlier studies, our data suggest that behavioural improvements already occur within the first 3 months after surgery. ${ }^{8}{ }^{10-12}$ Because there were no major changes in the circumstances and conditions of the children in the time before surgery, it is unlikely that variations in the time scale between presurgical evaluation and surgery (zero to 7 months) would have had any influence on our results.

The early changes in behaviour after epilepsy surgery raise the question as to which factors may have contributed to this finding.

Out of the analyzed variables with potential influence on behaviour only seizure outcome showed a significant relation with total behaviour changes. The better the postoperative seizure control, the more likely there were improvements in behaviour. It is interesting to note that other variables, which have been proved to influence behaviour in children with epilepsy, seemed to be irrelevant for changes in behaviour. ${ }^{67}$ Thus, the improvements in behaviour depended neither on age or sex of the patients nor on their presurgical conditions. In addition, our results also suggest that there are no differences between children with different focus sites. One of the most often suspected factors for improvements in postoperative behaviour - the reduction in antiepileptic medication-could also not be accounted for. According to the multivariate analysis of the total sample the changes in behaviour were not related to changes in antiepileptic treatment, thereby also indicating that the slight group differences in the antiepileptic treatment were not relevant for behavioural changes. Nevertheless, the data from the control group suggest that increases in the number of drugs may actually increase attention problems.

Some authors have emphasised the importance of psychosocial factors for the development of behavioural problems in children with epilepsy. ${ }^{72}$ We did not consider psychosocial variables in our present study, 
because we assume that important social factors (for example, family status) remained stable within the short follow up interval. Nevertheless, the successful surgical intervention may actually have changed some psychosocial conditions - for example, family stressand thereby may have positively influenced the child's social interactions with family members. This in turn may have led to increased self confidence, reduction of anxiety, and decline of aggressive behaviour in the young patient.

However, for at least three reasons we think that the behavioural improvements are rather due to biological factors than to psychosocial effects. Firstly, the behavioural changes occurred very early after the surgery and were extensive in some patients. Secondly, the social problems remained largely unchanged after surgery, and thirdly, the improvements were strongly related to decrement in attention problems. Attention deficit disorders in turn are widely accepted as the result of central nervous dysfunction. ${ }^{23}$ Evidence for this "biological hypothesis" comes also from findings in children with new onset epilepsies. Dunn et al found a high prevalence of behavioural disorders in children already before the first seizure. After successful antiepileptic treatment the behaviour problems diminished in many patients. ${ }^{20}$ The findings of Dunn et al and our own results suggest that the epileptic focus directly causes behavioural problems in children, which can be improved by efficient antiepileptic medication or surgical treatment of the epilepsy. This issue might be evaluated more extensively by analysis of the patient's preoperative and postoperative EEGs. However, for the purpose of the present study EEG data were not analyzed in detail.

A possible weakness of our study is the restriction of the child's examination to the rating of the parents. Parents whose children became seizure free might have extended the success of surgery in terms of seizure relief onto the child's behaviour, and thereby overestimated postoperative behaviour improvements (halo effect). Particularly in the first weeks after surgery the parents might be in a state of euphoria due to different factors, especially if seizure control has improved markedly. Avoiding such bias would require the evaluation of more objective data or the detailed observation by professional examiners, which is difficult to realise in outpatients. Therefore, the ratings of the parents currently seem to provide the best estimation of the child's behaviour and at least are superior to a global impression by the clinical examiner.

Finally, it should be pointed out that the aim of the study was to evaluate the behavioural development after surgical treatment in comparison with the development of non-operated paediatric patients with intractable epilepsies. Therefore our study was not designed to compare the behavioural effects of surgical versus drug treatment. Nearly all patients in both groups received antiepileptic drugs. Both groups were rather inhomogeneous in their drug regimen, but this factor was controlled within the statistical analyses and found to be of minor influence on the behavioural development. Nevertheless, the present evaluation referred to a very early postoperative follow up interval. Additional studies with longer follow up intervals should clarify if the achieved postoperative improvement in behaviour can be maintained or even continues in the long term development of the child.

In summary, the present data suggest that surgery in children with pharmacoresistant focal epilepsies is not only followed by successful seizure control, but is also accompanied by an early improvement of behavioural disorders. These behavioural improvements are assumed to result directly from the removal of the epileptic focus. They are not predictable on the basis of preoperatively available information and the site of surgery, but depend on the seizure outcome.

1 Rutter MJ, Tizard K, Whitmore. Education, health and behavior. London: Longmans, 1970.

2 McDermott S, Mani S, Krishnaswami S. A populationbased analysis of specific behavior problems associated with childhood seizures. $\mathcal{F}$ Epilepsy 1995;8:110-18.

3 Hoare P, Mann H. Self esteem and behavioral adjustment in children with epilepsy and children with diabetes. $\mathcal{F}$ Psychosom Res 1994;38:859-69.

4 Ounsted C. The hyperkinetic syndrome in epileptic children. Lancet 1955;2:303-11

5 Stores G. School children with epilepsy at risk for learning and behavioral problems. Dev Med Child Neurol 1978;20: $502-8$.

6 Hermann BP, Whitman S, Hughes JR, et al. Multietiological determinants of psychopathology and social competence in children with epilepsy. Epilepsy Res 1988;2:51-60.

7 Austin JK, Risinger MW, Beckett LA. Correlates of behavior problems in children with epilepsy. Epilepsia 1992;33: problems

8 Bourgeois M, Sainte-Rose C, Lellouch-Tubiana A, et al. Surgery of epilepsy associated with focal lesions in childhood. F Neurosurg 1999;90:833-42.

9 Aman MG, Werry JS, Turbott SH. Behavior of children with seizures. F Nerv Ment Dis 1992;180:124-9.

10 Lindsay J, Glaser G; Richards P, et al. Developmental aspects of focal epilepsies of childhood treated by neurosurgery. Dev Med Child Neurol 1984;26:574-87.

11 Davidson S, Falconer MA. Outcome of surgery in 40 children with temporal lobe epilepsy. Lancet 1975;i: 1260-3

12 Wilson PJE. Cerebral hemispherectomy for infantile hemiplegia. Brain 1970;93:147-80.

13 Verity CM, Strauss EH, Moyes PD, et al. Long term follow up after cerebral hemispherectomy: neurophysiologic, radiologic, and psychological findings. Neurology 1982;32: 629-39.

14 Lassonde M, Sauerwein C. Neuropsychological outcome of corpus callosotomy in children and adolescents. $\mathcal{F}$ Neurosurg Sci 1997;41:67-73.

15 Shorvon SD, Reynold EH. Reduction of polypharmacy for epilepsy. BMF 1979;ii:1023-5.

16 Cull CA, Trimble MR, Wilson J. Changes in antiepileptic drug regimen and behaviour in children with epilepsy. $\mathcal{F}$ Epilepsy 1992;5:1-9.

17 Achenbach TM, Edelbrock C. Manual for the child behavior checklist: 4-18 and 1991 profile. Burlington: University of Vermont, Department of Psychiatry, 1991.

18 Döpfner M, Schmeck K,Berner W. Handbuch: Elternfragebogen über das Verhalten von Kindern und fugendlichen. gen über das Verhalten von Kindern und fugendlichen.
Forschungsergebnisse zur deutschen Fassung der Child Behavior Forschungsergebnisse zur deutschen Fassung der Child Behavior
Checklist (CBCL/4-18). Köln: Arbeitsgruppe Kinder-, Checklist (CBCL/4-18). Köln: Arbeitsgruppe
Jugend- und Familiendiagnostik (KJFD), 1994.

19 Dorenbaum D, Cappelli M, Keene D, et al. Use of a child behavior checklist in the psychosocial assessment of children with epilepsy. Clin Pediatr (Phila) 1985;24:634-7.

20 Dunn DW, Austin JK, Huster GA. Behaviour problems in children with new-onset epilepsy. Seizure 1997;6:283-7.

21 Trimble MR. Antiepileptic drugs, cognitive function, and behavior in children: evidence from recent studies. Epilepsia 1990;31 (supp 4):30-4.

22 Hoare P, Kerley S. Psychosocial adjustment of children with chronic epilepsy and their families. Dev Med Child Neurol 1991;33:201-15.

23 Boliek CA, Obrzut JE. Neuropsychological aspects of attention deficit/ hyperactivity disorder. In: Reynolds CR, Fletcher-Janzen E, eds. Handbook of clinical child neuropsychology. New York: Plenum Press, 1997:619-33. 